\title{
The Plant Circadian Clock: From a Simple Timekeeper to a Complex Developmental Manager
}

\author{
Sabrina E. Sanchez and Steve A. Kay \\ Department of Cell and Molecular Biology, The Scripps Research Institute, La Jolla, California 92093 \\ Correspondence: stevekay@scripps.edu
}

The plant circadian clock allows organisms to anticipate the predictable changes in the environment by adjusting their developmental and physiological traits. In the last few years, it was determined that responses known to be regulated by the oscillator are also able to modulate clock performance. These feedback loops and their multilayer communications create a complex web, and confer on the clock network a role that exceeds the measurement of time. In this article, we discuss the current knowledge of the wiring of the clock, including the interplay with metabolism, hormone, and stress pathways in the model species Arabidopsis thaliana. We outline the importance of this system in crop agricultural traits, highlighting the identification of natural alleles that alter the pace of the timekeeper. We report evidence supporting the understanding of the circadian clock as a master regulator of plant life, and we hypothesize on its relevant role in the adaptability to the environment and the impact on the fitness of most organisms.

\begin{abstract}
A sessile organisms, plants cannot escape Afrom herbivores or elude unfavorable conditions. In addition, they have to cope with the constantly changing surroundings, for example, light-dark cycles imposed by the rotation of the planet. The circadian clocks enable plants to track those periodic changes, allowing them to anticipate and synchronize multiple physiological and developmental responses to the best time of the day or the year, which finally contributes to an enhanced fitness (Michael et al. 2003; Dodd et al. 2005; Greenham and McClung 2015).

Conceptually, the early understanding of the biological clock in plants suggested a linear or "stepwise" process in which environmental
\end{abstract}

signals (inputs) were responsible for synchronizing the endogenous timekeeper (the molecular mechanism tracking time), which then modulated several physiological responses such as leaf movement and gene expression (outputs) (Harmer 2009). Currently, the number of processes known to be regulated by the circadian oscillator has significantly expanded and, more surprisingly, most of these pathways feed back to modulate the function of the core clock itself. This review focuses on the model species Arabidopsis thaliana and provides an overview of the physiological pathways regulated by the biological clock and the feedback loops adjusting the endogenous timekeeper. In addition, we propose that the molecular pieces of the clockwork

Editors: Paolo Sassone-Corsi, Michael W. Young, and Akhilesh B. Reddy

Additional Perspectives on Circadian Rhythms available at www.cshperspectives.org

Copyright (C) 2016 Cold Spring Harbor Laboratory Press; all rights reserved; doi: 10.1101/cshperspect.a027748

Cite this article as Cold Spring Harb Perspect Biol 2016;8:a027748 
might act as hubs directly linking it to different processes of plant development. This intricate system led us to propose a model in which the core oscillator could function as a central regulator. For this, the information coming from the environment (external) and the endogenous status (internal) would be integrated, and a balanced output determined and translated to the many physiological and developmental traits this system modulates. This entire mechanism helps organisms to not only be synchronized with environmental stimuli and related changes, but also to determine the magnitude of different responses to better harness their resources, such as energy, to ensure survival and improve the fitness of the individual.

\section{CLOCK ORGANIZATION IN Arabidopsis thaliana}

A. thaliana is the model species used in almost every field of plant physiology and molecular biology research (Meinke et al. 1998; McClung 2013). Consequently, it is the organism in which the molecular mechanism underlying the endogenous clock is best described and has extensively been reviewed (Nagel and Kay 2012; Hsu and Harmer 2014). In this section, we will summarize the current knowledge for this model plant (Fig. 1).

The first molecular model of a biological timekeeper in plants was proposed in 2001 for A. thaliana, and was based on a negative transcriptional-translational feedback loop including three genes: two MYB transcription factors, LATE ELONGATED HYPOCOTYL and CIRCADIAN CLOCK ASSOCIATED 1 (LHY and CCA1, respectively), and TIMING OF CAB EXPRESSION 1 (TOC1) also known as PRR1 (PSEUDO-RESPONSE REGULATOR 1) (Alabadí et al. 2001). CCA1 and $L H Y$ are morning expressed genes. After translation, CCA1 and LHY mutually interact and repress TOC1 expression (Alabadí et al. 2001; Lu et al. 2009;

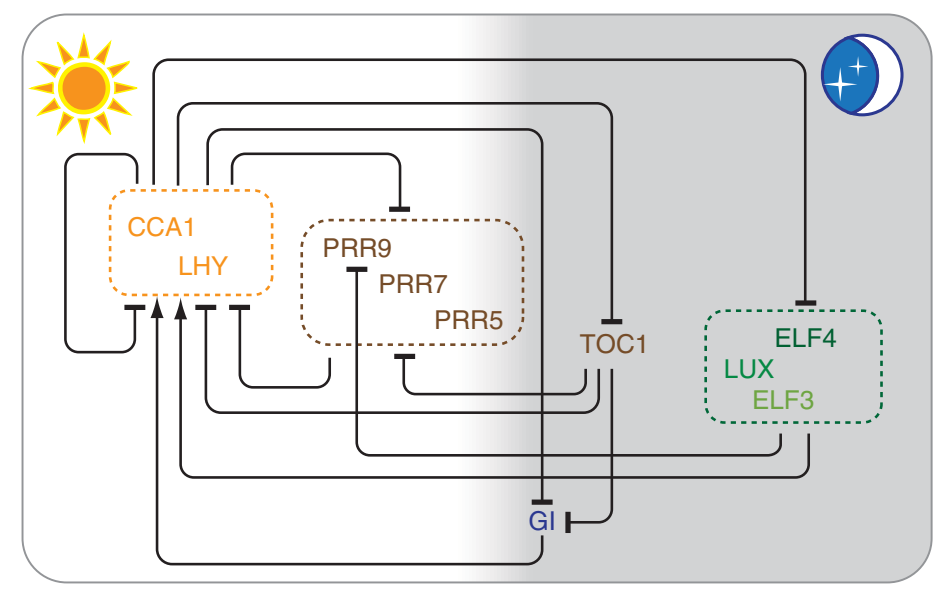

Figure 1. Minimal architecture underlying the circadian clock of Arabidopsis thaliana. In this simplified model, only a subset of the pieces of the trascriptional-translational feedback loops is shown. The clockwork components are represented from left to right according to the time-of-day of their peak expression. White and gray background depict day and night, respectively. CCA1 and LHY are expressed in the morning and repress the expression of all clock components represented here. The "evening complex" (EC) is formed by LUX, ELF3, and ELF4, and induces CCA1/LHY. PRRs are repressed by TOC1, and PRR9 is also negatively regulated by the EC. TOC1 represses GI, which in turn induces CCA1/LHY by an unknown mechanism. All members of the PRR family are known to negatively regulate CCA1/LHY. Rectangles denote "functional groups," either because the components are members of the same gene family (CCA/LHYand PRR9/PRR7/PRR5) or they act as a complex (EC). Despite PRR9, PRR7, and PRR5 being homologs of TOC1 (PRR1), the latter seems to have a different role in the oscillator. Arrowheads and perpendicular lines illustrate induction and repression of transcriptional activity, respectively. For references and a complete description, please refer to the main text. 
The Circadian Clock: A Master Regulator of Plant Life

Yakir et al. 2009). At dusk, CCA1 and LHY levels decrease; TOC1 is expressed and negatively regulates CCA1 and LHY transcription (Gendron et al. 2012; Huang et al. 2012). An additional loop is formed by PRR9, PRR7, and PRR5 (members of the $P R R$ gene family) that are expressed sequentially throughout the day, with PRR9 peaking in the morning (Nakamichi et al. 2005). These three genes show partially redundant functions, are homologs of TOC1, and repress CCA1 and $L H Y$ transcription $(\mathrm{Na}-$ kamichi et al. 2010). Genetic experiments suggest that $P R R 9$ and $P R R 7$ would be induced by CCA1 and LHY (Farré et al. 2005). However, recent evidence shows that LHY is, in fact, a direct repressor of these two genes, as well as PRR5 (Adams et al. 2015). In addition, they are also repressed by TOC1 (Huang et al. 2012). Furthermore, PRR9 expression is also repressed by a complex known as the "evening complex" (EC) (Nagel and Kay 2012). The EC is formed by the MYB-like transcription factor LUX ARRHYTHMO (LUX, also known as PHYTOCLOCK1), and EARLY FLOWERING 3 (ELF3) and ELF4, two nuclear proteins with unknown biochemical function (Nusinow et al. 2011). The EC and CCA 1 and $L H Y$ are reciprocally regulated with the EC indirectly promoting the expression of these two morning genes and, in turn, CCA1 and LHY repress the EC components (Nagel and Kay 2012; Adams et al. 2015). Furthermore, it has been shown that CCA1 and, LHY can bind to their own and each other's promoter to repress their transcription (Adams et al. 2015).

In recent years, a new loop was described encompassing a morning-expressed group of genes, REVEILLE (RVE), homologs of CCA1 and LHY. The novelty is that RVE8 induces the transcriptional activity of afternoon and evening-phased genes such as PRR5, TOC1, LUX, and ELF4 (Hsu et al. 2013). In turn, RVE8 is repressed by PRR9, PRR7, and PRR5. In addition, RVE6 and RVE4 appear to have partially redundant roles with their close relative RVE8 (Rawat et al. 2011; Nakamichi et al. 2012; Hsu et al. 2013; Hsu and Harmer 2014). Another small family of morning-expressed genes that plays an important role in the circadian oscillator is comprised by the NIGHT LIGHTINDUCIBLE AND CLOCK-REGULATED (LNK1, 2, 3, and 4) components (Rugnone et al. 2013). LNKs interact with the RVEs and have been shown to be able to either antagonize or coactivate RVE8 (Xie et al. 2014; Pérez-García et al. 2015).

CCA1, LHY, and TOC1 have also been shown to repress GIGANTEA (GI) expression, and in turn, GI induces CCA1 and LHY expression through an unknown mechanism (Park et al. 1999; Huang et al. 2012; Lu et al. 2012; Kim et al. 2013b; Adams et al. 2015). Although GI is not considered a core clock component, it might be an essential piece connecting the central oscillator with many physiological processes (Panigrahi and Mishra 2015). Recently, GI has been proposed as a hub regulating key features of plant life, such as flowering and salt and freezing tolerance, among others (Kazan and Lyons 2015; Panigrahi and Mishra 2015).

In addition to the transcriptional-translational feedback loops described above (and all the additional components not mentioned in this review), the biological timekeeper owes its robustness to many other mechanisms, including epigenetic, posttranscriptional, and posttranslational regulation (Seo and Mas 2014; Nolte and Staiger 2015; Romanowski and Yanovsky 2015). Communication between organelles (nucleus-chloroplast) also contributes to the proper ticking of the clock (Hassidim et al. 2007). Furthermore, similar to what is known in animals, Arabidopsis might also contain tissuespecific clocks (James et al. 2008; Allada and Chung 2010; Mohawk et al. 2012; Endo et al. 2014; Takahashi et al. 2015). Nevertheless, the interaction and the hierarchical structure between these timekeepers remain to be clarified.

\section{THE CLOCK WIRING IN Arabidopsis thaliana}

The biological clock modulates a myriad of plant responses referred to as "outputs." Most of these processes feed back to the central oscillator, creating a complex web of connectivity. High-throughput assays have allowed us to determine the extent of the pervasiveness of the clock on gene expression (Harmer et al. 2000; 
Nagel et al. 2015), and multiple studies continue to confirm the impact of this regulation in different processes of plant physiology.

Two of the earliest and best-characterized outputs are photoperiodic flowering and growth of the seedling stem (hypocotyl). Both of these processes require the appropriate coincidence between internal rhythms, driven by the endogenous clock, and external conditions guided by photoperiod. However, the molecular mechanisms underlying these responses have been described and extensively reviewed (Greenham and McClung 2015), and therefore will not be discussed further in this review. Instead, we attempt to briefly describe some other key connections established by the clock.

\section{METABOLISM}

\section{Carbohydrates}

Photosynthesis enables plants to obtain energy and the structural components needed for growth and development from sunlight. Different steps of this process are circadian regulated (Harmer et al. 2000; Dodd et al. 2005; Noordally et al. 2013; Haydon et al. 2015). During the Calvin cycle, atmospheric carbon dioxide is assimilated by the enzyme ribulose-1,5-bisphosphate carboxylase/oxygenase (Rubisco). This protein is composed of several subunits and, to be functional, it needs to be assembled and activated (Andersson and Backlund 2008). The genes RCA (RUBISCO ACTIVASE) and at least one of the components of the RUBISCO SMALL SUBUNIT (RCBS) gene family have a circadian profile of expression, with a peak after dawn (Pilgrim and McClung 1993). In addition, the messenger RNA of $R C A$ is alternatively spliced, giving rise to two proteins with different regulatory features depending on light intensity (Zhang et al. 2002). Considering that the ratio between these isoforms has also been shown to follow a circadian rhythm (Sanchez et al. 2010), this regulation could be hypothesized to have a physiological relevance. This suggests that the clock contributes to the fine-tuning of this efficient system, providing the tools to better respond to the variable light conditions throughout the day.
The thioredoxin family of proteins is essential in the redox balance that enables carbon assimilation, and constitutes another example of the control imposed by the endogenous oscillator on carbohydrate metabolism. The thioredoxin $f$ and $m$ show a circadian profile of expression and biochemical assays suggest that CCA1 might be directly involved in that regulation (Barajas-López et al. 2011).

During the day, carbon is assimilated as glucose, then converted to sucrose for transport and stored as starch, which is gradually consumed during the night (Smith and Stitt 2007). Despite the fact that the detailed mechanism is unknown, it has been shown that the linear and appropriate starch degradation during the night requires a functional circadian clock (Graf et al. 2010). In seedlings, it has been established that stem elongation is rhythmic with maximal growth at the end of the night (Nozue et al. 2007). Therefore, it is likely that failure to adjust the proper rate of starch degradation during the night might result in carbon starvation before dawn with the consequent reduction of growth (Graf et al. 2010).

High-throughput expression analysis showed that glucose modulates many circadian-regulated genes in carbon-starved seedlings, suggesting that sugar could be an input for the core oscillator (Bläsing et al. 2005). Supporting this idea, exogenous sucrose has the ability to shorten the period in wild-type plants grown under free-running conditions (Knight et al. 2008). In addition, the sensitivity of the circadian clock to sugar was also reported, and these studies showed that exogenous sucrose can modify properties of the circadian oscillator such as amplitude and period, as well as entrain the promoter activity of several core clock genes under continuous dark (Dalchau et al. 2011). Interestingly, genetic experiments revealed that $G I$ is necessary for the proper clock response to sugar and its own expression is modulated by sucrose (Dalchau et al. 2011). Finally, Webb and colleagues have presented very convincing evidence suggesting that the endogenous clock is entrained by photosynthetic cues (Haydon et al. 2013). In that study, the investigators showed that metabolically active sugars (sucrose, fruc- 
The Circadian Clock: A Master Regulator of Plant Life

tose, and glucose but not mannitol) are able to entrain and modify the phase of the core oscillator. Although the inhibition of photosynthesis modifies the pace of the clock, the normal period can be reestablished by the exogenous addition of sucrose. The clock gene PRR7 was shown to be an important link in the connection between sugar and the central oscillator, although CCA1 and LHY might also be implicated in the pathway (Haydon et al. 2013). In summary, carbohydrates can influence the biological timekeeper by regulating the transcriptome and the period or the phase of the clock under different growing conditions. Considering that sugar could contribute to the fine-tuning of the circadian clock and that flowering time in long days is tightly controlled by the endogenous oscillator, it has been hypothesized that carbohydrates could also have a role in modulating the sensitivity to photoperiodic detection (Dodd et al. 2015).

\section{Mineral Nutrients}

Nutrient elements and ions play numerous roles in plant metabolism. They can act as cofactors or signaling molecules, and are required for protein and macromolecule biosynthesis. Both micro (e.g., copper, iron, zinc, manganese) and macronutrients (i.e., nitrogen, phosphorous, sulfur, calcium, magnesium, potassium) are essential, but in precise amounts. Deficiency impacts plant growth and excess may be toxic (Haydon et al. 2015). Nutrient demand follows a rhythmic pattern as a consequence of diel changes, for instance, in photosynthesis and transpiration rates, suggesting they are under a tight metabolic and clock control (Fig. 2A) (Haydon et al. 2015).

\section{Nitrogen}

Nitrogen $(\mathrm{N})$ is an essential constituent of nucleotides and proteins. Downstream products from $\mathrm{N}$ assimilation (organic $\mathrm{N}$ ) can modulate the inorganic $\mathrm{N}$ uptake by different mechanisms (Xu et al. 2012). Gutiérrez et al. (2008) showed that organic $\mathrm{N}$ is able to modify CCA1 expression levels and to induce phase-shifts in the circadian clock, suggesting that organic $\mathrm{N}$ can act as an entrainment signal. Conversely, CCA1 transcriptionally regulates genes downstream in the $\mathrm{N}$ metabolism by binding their promoters (Gutiérrez et al. 2008). This evidence supports CCA1, a core clock protein, as the main candidate linking the circadian oscillator with nitrogen metabolism.

\section{Calcium}

Calcium $\left(\mathrm{Ca}^{2+}\right)$ participates in cell signaling as a secondary messenger (Dodd et al. 2010). Cytosolic concentration of free $\mathrm{Ca}^{2+}\left(\left[\mathrm{Ca}^{2+}\right]_{\text {cyt }}\right)$ is rhythmic (Johnson et al. 1995; Love et al. 2004) and most likely under the control of the circadian clock (Love et al. 2004), providing a potential mechanism for the biological oscillator to modulate some of its outputs. Furthermore, transcripts of genes coding for several channels and transporters related to $\mathrm{Ca}^{2+}$ metabolism have been shown to be clock regulated (Haydon et al. 2011, 2015). cADPR is a cytosolic metabolite involved in $\mathrm{Ca}^{2+}$ homeostasis and its concentration is circadian regulated (Dodd et al. 2007). Nicotinamide inhibits the synthesis of cADPR and abolishes the $\left[\mathrm{Ca}^{2+}\right]_{\text {cyt }}$ oscillations, proposing that the core clock might modulate $\left[\mathrm{Ca}^{2+}\right]_{\text {cyt }}$ rhythms, at least partially, by regulating cADPR (Dodd et al. 2007). Using transgenic plants and microarray experiments, the expression of GI, PRR5, PRR7, LHY, and CCA1, among others, was shown to be controlled by cADPR (Sánchez et al. 2004; Dodd et al. 2007), suggesting a reciprocal regulation.

\section{Iron}

Iron $(\mathrm{Fe})$ can change the oxidation state and participates in many metabolic processes involving electron transfer reactions. In plants, Fe has a major role in photosynthesis, which in turn impacts carbon assimilation and biomass production (Ravet et al. 2009; Gayomba et al. 2015). On the other hand, Fe ions promote oxidative stress that can be toxic for cells (Gayomba et al. 2015). Therefore, it is crucial for the organism to possess tightly regulated and coordinated responses to cope with either 
A

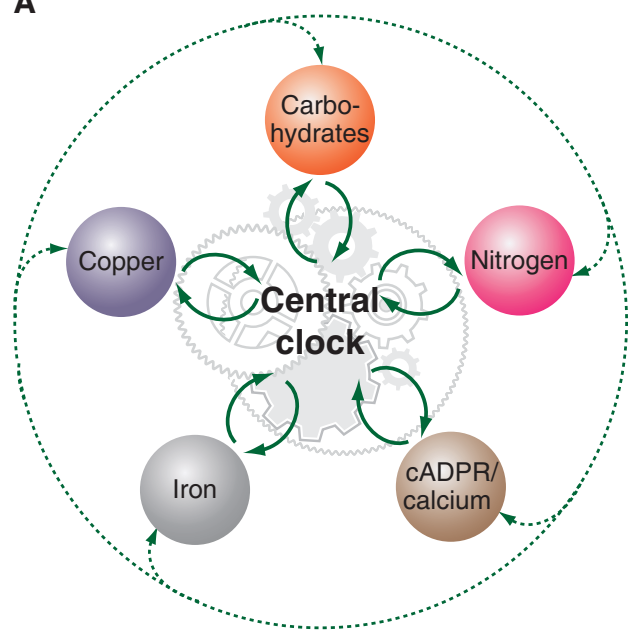

B

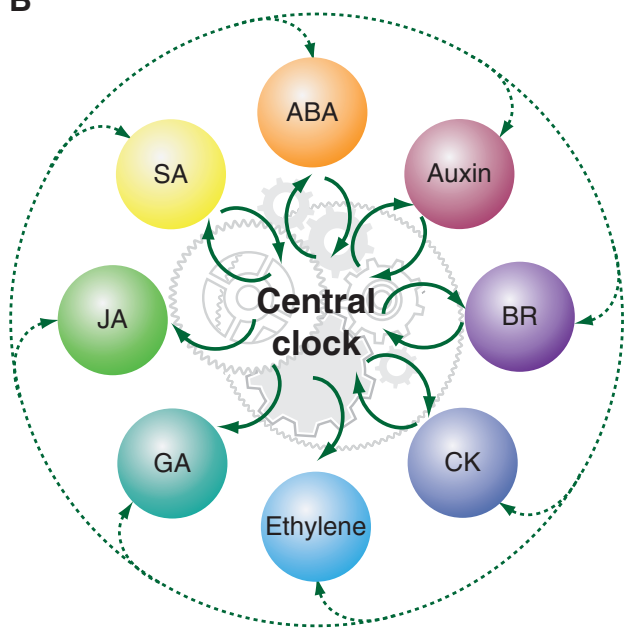

Figure 2. Interlocked communication between the central clock and different plant pathways. $(A)$ The central clock modulates carbohydrate metabolism, nitrogen, calcium, iron, and copper homeostasis. In turn, all metabolic processes involving these nutrients feed back to the core oscillator. $(B)$ The central clock modulates the hormone pathways illustrated in the figure: ABA, abscisic acid auxin; BRs, brassinosteroids; CK, cytokinin; ethylene; GA, gibberellin; JAs, jasmonates; SA, salicylic acid. All of these hormones with the exception of ethylene, GA, and JA contribute to the fine-tuning of the timekeeper. The outer arrows and circle reflect the clock independent interaction of these plant pathways, adding further complexity to the system as a whole.

iron deficiency or overload. The messenger RNA of FER1, a gene encoding an Fe storage protein located in plastids, oscillates with a period of $24 \mathrm{~h}$ and that rhythm is abolished in different core clock gene mutant backgrounds. FER1, as well as other members of the gene family, and Fe transporters are circadian regulated and repressed by TIC (TIME FOR COFFEE) (Duc et al. 2009; Hong et al. 2013), a nuclear protein able to modulate the clock function (Hall et al. 2003; Ding et al. 2007). Moreover, PRR7 binds to Fer1, Fer3, and Fer4 promoters (Liu et al. 2013), strengthening the notion that iron homeostasis is regulated by the biological timekeeper. This might reflect biological relevance, because both tic mutants and PRR7 overexpressor lines show phenotypes associated with inefficient regulation of Fe (Duc et al. 2009; Liu et al. 2013).

In 2013, three independent studies showed that Fe-deficiency slows down the pace of the core clock (Chen et al. 2013; Hong et al. 2013; Salomé et al. 2013). Functional chloroplasts, but not photosynthetic activity, were shown to be necessary for the period lengthening induced by Fe deficiency (Chen et al. 2013; Salomé et al. 2013). Although CCA1 and $L H Y$ are necessary for the signaling of Fe deficiency to the core clock, there is contradictory evidence for the role of GI and ZTL in this process (Chen et al. 2013; Hong et al. 2013; Salomé et al. 2013). These compelling data supports a mechanism for Fe homeostasis and the circadian clock being reciprocally regulated. Interestingly, retrograde signals ("message" from chloroplast to nucleus) synchronizing the core clock in response to Fe deficiency, constitute the second evidence of the role of this organelle as a timer for the central oscillator (Hassidim et al. 2007; Chen et al. 2013; Salomé et al. 2013).

\section{Copper}

Copper $(\mathrm{Cu})$ is a cofactor involved in redox pathways and also has an effect on the ticking of the biological clock. Despite the fact that the connection appears elusive, it has been shown that addition of $\mathrm{Cu}$ in the growth medium 
The Circadian Clock: A Master Regulator of Plant Life

dampens the amplitude of oscillation of CCA1 and LHY transcripts (Andrés-Colás et al. 2010), as well as GI transcriptional activity (Perea-García et al. 2015). A phase response curve (adding $\mathrm{Cu}$ at different moments of the day) evidenced that this metal can modify the phase and amplitude of $L H Y$ promoter activity, although that response is not gated by the endogenous clock (Andrés-Colás et al. 2010). However, the period of core clock transcripts does not seem to be altered neither by the excess nor the deficiency of Cu (Andrés-Colás et al. 2010; Chen et al. 2013; Salomé et al. 2013; Perea-García et al. 2015). Recently, genes regulating $\mathrm{Cu}$ homeostasis have been shown to follow a circadian pattern (Perea-García et al. 2015), suggesting a reciprocal interaction. To fully understand whether the responses observed on increased $\mathrm{Cu}$ levels are a direct effect on the circadian oscillator or simply the consequence of altering the general metabolic status of the cell, for example, by modifying its redox balance or hormonal pathways (Peñarrubia et al. 2015), more experiments need to be performed.

\section{Summary}

The mechanisms by which developmental and physiological responses are modulated by the availability of a particular metal are not known (Chen et al. 2013). Considering the ability of several nutrients to regulate the ticking of the core clock, it is tempting to speculate that the biological oscillator could be the link between the metal status and the metabolic pathways controlling the general plant responses.

\section{HORMONE PATHWAYS}

Plant hormones (phytohormones) are small signaling molecules that are able to orchestrate multiple cellular processes that impact physiology and growth (de Lucas and Prat 2014; Larrieu and Vernoux 2015). Notably, a single hormone can modulate many responses, whereas several hormones can regulate a specific process. For such a complex system to work, a delicate cross talk among these endogenous molecules is necessary. Furthermore, different hormone pathways interact with the circadian oscillator, increasing the complexity of the network (Fig. 2B) (Robertson et al. 2009; Seung et al. 2012; de Lucas and Prat 2014; Peñarrubia et al. 2015).

In 2008, Covington et al. (2008) showed that transcript levels of hormone-responsive genes were circadian regulated, suggesting that the endogenous oscillator was either modulating the hormone levels and/or the activities within the signaling pathway for most of the phytohormones. The circadian regulation of several hormone signaling pathways was further confirmed by an array of other studies (Thain et al. 2004; Bancos et al. 2006; Covington and Harmer 2007; Mizuno and Yamashino 2008; Arana et al. 2011; Shin et al. 2012; Wang et al. 2014; Zheng et al. 2015). Furthermore, diel oscillations in phytohormone abundance have been determined (Thain et al. 2004; Nováková et al. 2005; Robertson et al. 2009). Conversely, exogenously added cytokinin, auxin, brassinosteroids (BR), or abscisic acid (ABA), modifies at least one clock parameter, indicating that these molecules modulate the core oscillator activity (Hanano et al. 2006). Several hormone mutants also display clock phenotypes (Hanano et al. 2006), reinforcing the idea that hormone pathways feed back to the molecular clock. Nevertheless, experiments with gibberellin (GA) (Hanano et al. 2006; Arana et al. 2011) and ethylene (Thain et al. 2004; Hanano et al. 2006) showed subtle or no effect on circadian clock functioning. Finally, whereas the role of jasmonates (JA) remains to be elucidated, the evidence suggests that salicylic acid (SA) modulates the oscillator activity, although through a more elusive pathway (Fig. 2B) (Hanano et al. 2006; Zhou et al. 2015).

$\mathrm{ABA}$ regulation constitutes a good example of the many processes coordinated by a single molecule, and the numerous cross talk that occurs to achieve a balanced response and proper development of the organism. This phytohormone, known as one of the canonical "stress hormones," regulates, for example, seed germination, stomatal opening, osmotic stress tolerance, and pathogen attack responses (Seung et al. 2012). ABA connection with the circadian clock has also been established. Clock regula- 
tion of genes involved in different steps of ABA synthesis (Covington et al. 2008; Fukushima et al. 2009) and rhythmic diel levels of ABA in leaves have been reported (Lee et al. 2006). Furthermore, ABA-responsive genes are also circadian regulated, either as direct targets of the central clock or as a response of the rhythmic ABA signaling network (Seung et al. 2012; Liu et al. 2013). For example, TOC1 binds to $A B A R$ ( $A B A$-related gene) promoter. This gene is one of the putative ABA receptors and TOC1 binding is necessary for its circadian profile of expression (Legnaioli et al. 2009). This suggests that TOC1 modulates the sensitivity to ABA. Surprisingly, ABA acutely induces TOC1 expression in a gated fashion, and this regulation depends on a functional ABAR (Legnaioli et al. 2009), closing an elegant feedback loop ABACLOCK-ABA.

There is also cross talk between ABA and $\mathrm{Ca}^{2+}$ metabolism. ABA induces the activity of ADPR (ADP-ribosyl cyclase), the enzyme responsible for cADPR synthesis, and cADPR up-regulates the expression of ABA-related genes (Sánchez et al. 2004). As explained in the previous section, CADPR is a metabolite involved in $\mathrm{Ca}^{2+}$ homeostasis, and it is known to be a target as well as a regulator of the circadian clock (Dodd et al. 2007). Therefore, ABA metabolism is associated with $\mathrm{Ca}^{2+}$ homeostasis and the circadian clock. Importantly, this regulatory network leads the stomatal opening to follow a circadian profile (opening at dawn and closing before evening), and therefore also implicates a link to drought tolerance (Seo and Mas 2015).

\section{BIOTIC AND ABIOTIC STRESSES}

As sessile organisms, plants have to cope with different stresses, either biotic or abiotic, from which they cannot escape. Hormone signaling, followed by a rearrangement of the transcriptome and metabolism, is necessary to overcome these unfavorable conditions. More recently, the circadian clock has been shown to play an important role in the proper establishment of these responses and has been extensively reviewed (see Bolouri Moghaddam and Van den Ende
2013; Goodspeed et al. 2013; Greenham and McClung 2015; Grundy et al. 2015; Seo and Mas 2015).

\section{Abiotic Stress}

The most common abiotic stresses encompass drought, high and low temperature, and salinity. As mentioned above, in Arabidopsis, ABA plays an important role in drought responses due to its cross talk with calcium metabolism and the control of stomata opening and closure. In addition, the plant responses to drought are modulated by the biological oscillator, which acts through different components. As explained above, TOC1 forms a regulatory feedback loop with the ABA pathway, and drought-responsive genes have been shown to be negatively controlled by PRR7, through a TOC1-independent mechanism. Furthermore, tic mutants show an enhanced tolerance to drought, suggesting that this clock component is either directly or indirectly associated to this phenotype (Sanchez-Villarreal et al. 2013). In terms of salt tolerance, GI appears to be the molecular link between the clock and the triggered responses (Kim et al. 2013a), although PRR7 might also be involved in this mechanism (Kolmos et al. 2014).

The canonical response to cold temperatures is mediated by the $C B F / D R E B 1$ ( $C$ REPEAT BINDING FACTOR/DEHYDRATION-RESPONSIVE ELEMENT-BINDING) family of genes. CBFs activate the expression of the COLD-REGULATED (COR) genes, and are also involved in drought-tolerance responses. CBFs are clock regulated, therefore, their circadian oscillation is abolished in clock mutants and their targets also follow a diel profile of expression (Covington et al. 2008; Dong et al. 2011; Liu et al. 2013). Several clock mutants have also been shown to have cold phenotypes, suggesting the relevance of the timekeeper in establishing a proper response in this pathway (Nakamichi et al. 2009; Dong et al. 2011; Chow et al. 2014). In contrast, it is known that cold responses feed back to the central oscillator and dampen the amplitude of several core clock genes. For example, CBF1 binds to 
The Circadian Clock: A Master Regulator of Plant Life

the LUX promoter and regulates its expression, providing a possible molecular mechanism for the feedback loop (Chow et al. 2014). Moreover, cold temperatures can modulate alternative splicing of CCA1 and other clock genes, which impacts the proper functioning of the timekeeper (Grundy et al. 2015). The central oscillator is also modulated by high temperatures, either by regulation of the alternative splicing of clock components (Grundy et al. 2015) or through direct transcriptional regulation of them: HsfB2b (a heat shock factor) and FLOWERING BASIC HELIX-LOOP-HELIX 1 (FBH1), bind to PRR7 and CCA1 promoters, respectively (Kolmos et al. 2014; Nagel et al. 2014).

\section{Biotic Stress}

JA and salicylate (SA) are key phytohormones in the biotic stress-response pathways. Essentially, JA is associated with defense responses against herbivore attacks, whereas SA is implicated against pathogens. The signaling pathways for JA and SA are connected and show an antagonistic role on each other (Smith et al. 2009). In recent years, several publications revealed the importance of the core clock in the regulation of these two hormones, and therefore the responses they control. JA and SA accumulation patterns follow a circadian profile but with opposite phases. The timing of JA accumulation seems to be physiologically relevant, because its phase anticipates the peak of the insect feeding behavior, and a functional clock was shown to be required for this response (Goodspeed et al. 2012, 2013). In addition, JA signaling also contributes to the rhythmic susceptibility of A. thaliana to Botrytis cinerea, a necrotrophic fungal pathogen (Ingle et al. 2015). The clock gene TIC has been shown to be involved in the regulation of the JA receptor CORONATINEINSENSITIVE 1 (COI1) and MYC2, a bHLH transcription factor that induces the transcription of JA-responsive targets (Shin et al. 2012). Hence, TIC might represent the molecular link between the core oscillator and JA signaling.

SA responses also seem to depend on a functional clock. Biosynthesis of SA, and therefore SA accumulation, are regulated by CCA1
HIKING EXPEDITION (CHE), a core clock component (Zheng et al. 2015). Furthermore, CCA1 was shown to directly regulate PHT4; 1 , a phosphate transporter involved in SA-mediated defenses (Wang et al. 2014). Altogether, these data suggest that the biological oscillator is required for the establishment of proper responses against biotic stresses. However, the molecular understanding of this regulatory network is limited and requires further studies.

\section{THE CLOCK AS THE "MASTERMIND” OF PLANT LIFE}

In the previous sections, we outlined the molecular basis of the endogenous biological clock and highlighted its relevance in A. thaliana development and physiology. The processes controlled by the clock, as well as the molecular structure and several components of the core oscillator are conserved across different groups in the green lineage, including angiosperms and gymnosperms (Farré and Liu 2013; McClung 2013; Bendix et al. 2015). Agricultural traits, such as flowering time and light responses, have been extensively studied and shown to be under circadian clock regulation in different species, including many crops (Bendix et al. 2015). A clear example of the significant role the pacemaker plays in plant performance has recently been described in tomato (Müller et al. 2015). In this study, the investigators showed that diverse accessions of wild and cultivated tomato show different period lengths as a consequence of carrying distinct natural alleles of a gene involved in clock regulation. In addition, cultivated tomatoes grown in long days show an enhanced performance compared with wild relatives grown in long days. This different phenotype might be the result of the resonance between the pace of the endogenous clock and the environmental cues (Müller et al. 2015). This work suggests that human selection of a natural allele led to the enhancement of the crop performance under certain environmental conditions through alteration of the biological clock. It also supports the longstanding idea that manipulating the clock can increase yield and improve other agricultural traits. 
Surprisingly, despite the relevance of the pacemaker in governing the fate of plant life, proper functioning of the timekeeper is not required for the survival of the organism. It has been described that plants with an arrhythmic or desynchronized oscillator are still able to complete their life cycle (Hazen et al. 2005; Okada et al. 2009; Campoli et al. 2013). For example, as mentioned before, TIC is a circadian regulator related to iron homeostasis and JA signaling. Moreover, transcriptomic, metabolic and physiological analyses established that tic mutants show developmental, metabolic, and stress-related phenotypes (Sanchez-Villarreal et al. 2013). However, these plants are able to complete their life cycle and produce offspring. It is important to mention that some plant species (i.e., tomato and eggplant) show negative effects, such as leaf chlorosis and necrosis, when grown under continuous light that leads to decreased yield (Velez-Ramirez et al. 2011, 2014). The asynchrony between the endogenous clock and the external conditions has been proposed as one of the several components underlying this behavior (Velez-Ramirez et al. 2011).

Examining the evidence supporting the wiring of the oscillator to virtually every response of the organism, we propose that the central clock could be considered as a master regulator of plant growth, development, and physiology, providing not only time prediction features, but also adaptability to changing environmental conditions (Fig. 3). In this model, as a consequence of the perception of the surrounding cues and endogenous signaling, the core oscillator is fed with information coming from many different plant processes (e.g., metabolism and light signaling). This data is integrated and feedback messages are sent to establish the behavior ("output") of the organism. The final phenotype is modulated by clock-independent interactions of these different pathways. The continuous feedback loops and the many layers of communication provide robustness and flexibility to the endogenous clock. This mechanism can influence nutrient homeostasis, energy metabolism, and water usage to achieve an optimized balance that maximizes the fitness of the organisms and ensures the survival of the species. Although the idea of the clock as a main hub has been previously suggested (PereaGarcía et al. 2010; Pruneda-Paz and Kay 2010; Fogelmark and Troein 2014; Greenham and McClung 2015; Peñarrubia et al. 2015) continuous evidence over the years has greatly enriched and reinforced this notion.

For example, the clock seems to play an essential role in the classic trade-off between growth and defense (Huot et al. 2014) by timing immunity to the morning (Wang et al. 2011) and growth to the night (Nozue et al. 2007; Nusinow et al. 2011). Activation of plant defenses has a cost for growth and fitness (Huot et al. 2014). Data show that perturbation of the oscillator might induce an enhanced plant response to pathogens but also results in less growth (Zhou et al. 2015). In addition, the effect on the fitness costs has been proved to be different depending on the time of day that the pathogen attack occurs (Baldwin and Meldau 2013). This suggests that clock regulation of these mechanisms might represent an adaptive advantage and be related to the fitness of the organism.

It is tempting to speculate that the role of the biological clock might be more relevant under unfavorable conditions, when resources are limiting and the system is really challenged. The tight regulation and the effective communication conferred by the presence of a clockwork would provide an increased adaptability of the organism to the environment. Perhaps this system is not required for a single organism to survive but represents a significant adaptive advantage by improving the fitness of all individuals, which could then impact the evolution of the species. To test this hypothesis, long-term studies aimed at understanding the population dynamics of plants with a defective clock need to be explored.

\section{CONCLUDING REMARKS}

Manipulating the central oscillator to enhance crop yield and traits of agronomic interest has been suggested for a long time (Bendix et al. 2015). However, a key limitation in achieving this goal is that engineering the clockwork 


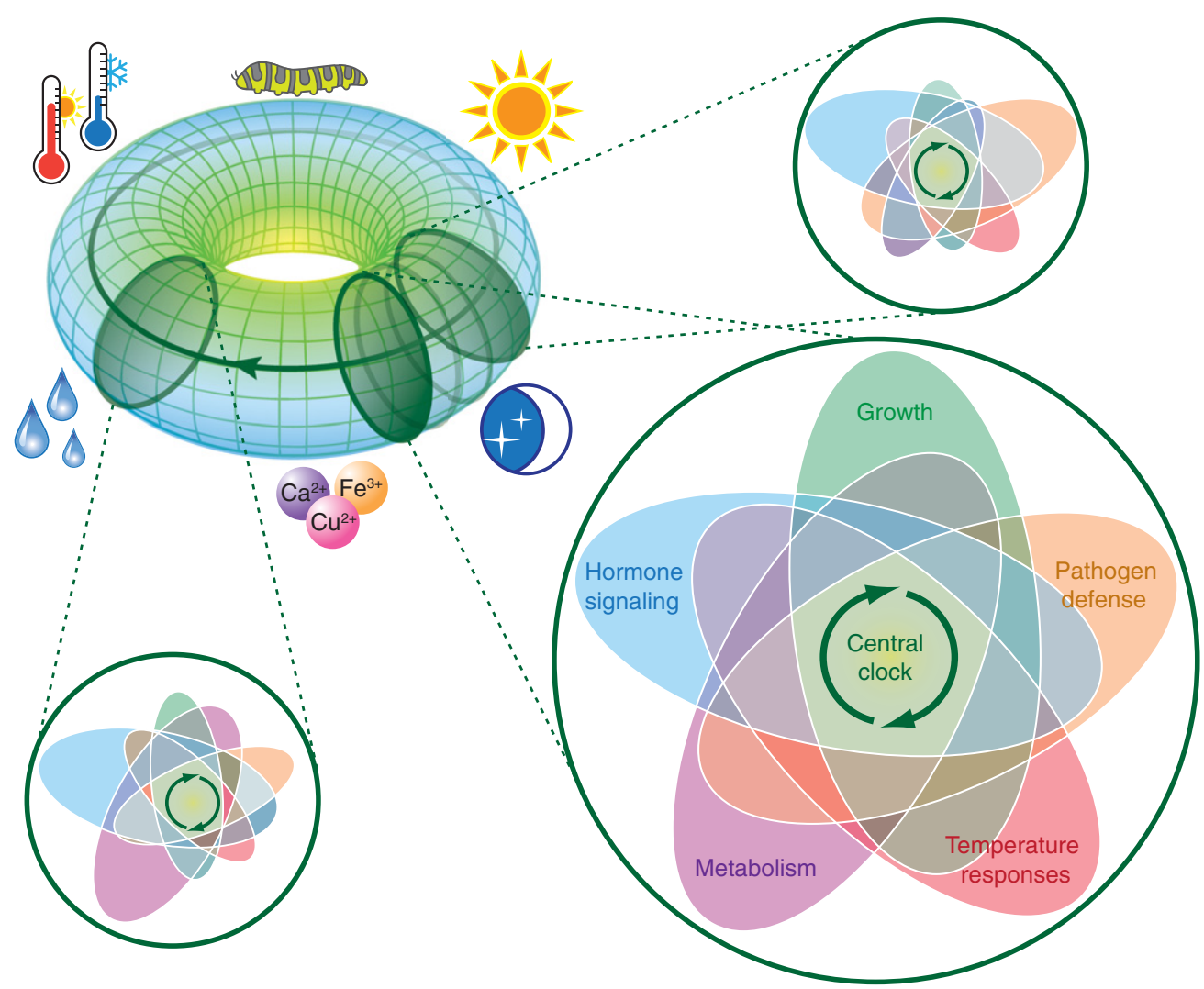

Figure 3. Schematic model of the circadian clock as the master regulator of plant life. The toroid represents all of the possible combinations of environmental parameters a plant can encounter through its life cycle. It comprises seasonal and diel changes of temperature and light, heat or cold stresses, water and mineral availability, and the presence of pathogens or herbivores, among others. Each slice of the toroid is therefore one particular combination of these external cues. For example, the bottom-right circle illustrates one hypothetical scenario. The different ellipses represent specific plant processes that interact with each other, converging in the center to feed the clock with exogenous and endogenous information, contributing to its synchronization. The central oscillator "interprets and translates" that information to determine an integrative and balanced response, which is then transmitted to the "outputs." The interaction between the core clock and the outputs establishes the plant phenotype, represented in the figure by the edge of the circle. The two smaller circles depict other environmental interactions and scenarios. As the "input" cues differ in each example, the outcome is also different. In these examples, the change in the size of the ellipses represents that under certain conditions some physiological pathways are prevalent over others.

might lead to not only the expected response, but also unexpected interactions and behavior. Furthermore, due to the tangled connections and feedback loops of the endogenous clock, it is hard to intuitively predict the resultant phenotype after perturbation (Fogelmark and Troein 2014). Contributions from mathematical models and system-level approaches that have already proved to be useful (Gutiérrez et al. 2008; Pokhilko et al. 2012; Fogelmark and Troein 2014; Zhou et al. 2015) might help to pave the way for this undertaking.

These methodologies could allow researchers to predict how a plant will behave under a certain set of environmental conditions, saving a lot of time and effort compared with generating a transgenic organism and testing it in the field (Ruffel et al. 2010). To build accurate 
mathematical models and to apply integrative system-level approaches, a solid and comprehensive knowledge of the clockwork is required. To determine clock features such as the period, it is necessary to perform experiments under continuous conditions. However, data of the biological oscillator behavior under set-ups resembling natural circumstances (i.e., lightdark cycles) are limited. The upcoming challenge for plant circadian clock research is performing studies under such conditions, which better mimic the natural scenarios. These approaches will improve our understanding of the biological clock, and therefore help us to better predict how a modified genome would respond to diverse and dynamic environmental cues.

\section{ACKNOWLEDGMENTS}

The authors thank D. H. Nagel, M. A. Nohales, and M. J. Yanovsky for critical reading of the manuscript. The authors apologize for not citing all relevant publications by coworkers because of space limitations. S.E.S. is partially supported by a postdoctoral fellowship from the Pew Latin American Fellows Program. This work is supported by the National Institute of General Medical Sciences of the National Institutes of Health (NIH) under award Nos. RO1GM067837 and RO1GM56006. The content is the sole responsibility of the authors and does not necessarily represent the official views of the NIH. This is manuscript \#29308 from The Scripps Research Institute.

\section{REFERENCES}

Adams S, Manfield I, Stockley P, Carré IA. 2015. Revised morning loops of the Arabidopsis circadian clock based on analyses of direct regulatory interactions. PLoS ONE 10: e 0143943 .

Alabadí D, Oyama T, Yanovsky MJ, Harmon FG, Mas P, Kay SA. 2001. Reciprocal regulation between TOC1 and LHY/ CCA1 within the Arabidopsis circadian clock. Science 293: 880-883.

Allada R, Chung BY. 2010. Circadian organization of behavior and physiology in Drosophila. Annu Rev Physiol 72: 605-624.

Andersson I, Backlund A. 2008. Structure and function of Rubisco. Plant Physiol Biochem 46: 275-291.
Andrés-Colás N, Perea-García A, Puig S, Peñarrubia L. 2010. Deregulated copper transport affects Arabidopsis development especially in the absence of environmental cycles. Plant Physiol 153: 170-184.

Arana MV, Marín-de la Rosa N, Maloof JN, Blázquez MA, Alabadí D. 2011. Circadian oscillation of gibberellin signaling in Arabidopsis. Proc Natl Acad Sci 108: 9292-9297.

Baldwin IT, Meldau S. 2013. Just in time: Circadian defense patterns and the optimal defense hypothesis. Plant Signal Behav 8: e24410.

Bancos S, Szatmári AM, Castle J, Kozma-Bognár L, Shibata K, Yokota T, Bishop GJ, Nagy F, Szekeres M. 2006. Diurnal regulation of the brassinosteroid-biosynthetic CPD gene in Arabidopsis. Plant Physiol 141: 299-309.

Barajas-López JdeD, Serrato AJ, Cazalis R, Meyer Y, Chueca A, Reichheld JP, Sahrawy M. 2011. Circadian regulation of chloroplastic $\mathrm{f}$ and $\mathrm{m}$ thioredoxins through control of the CCA1 transcription factor. J Exp Bot 62: 2039-2051.

Bendix C, Marshall CM, Harmon FG. 2015. Circadian clock genes universally control key agricultural traits. Mol Plant 8: $1135-1152$.

Bläsing OE, Gibon Y, Gunther M, Hohne M, Morcuende R, Osuna D, Thimm O, Usadel B, Scheible WR, Stitt M. 2005. Sugars and circadian regulation make major contributions to the global regulation of diurnal gene expression in Arabidopsis. Plant Cell 17: 3257-3281.

Bolouri Moghaddam MR, Van den Ende W. 2013. Sweet immunity in the plant circadian regulatory network. J Exp Bot 64: 1439-1449.

Campoli C, Pankin A, Drosse B, Casao CM, Davis SJ, von Korff M. 2013. HvLUX1 is a candidate gene underlying the early maturity 10 locus in barley: Phylogeny, diversity, and interactions with the circadian clock and photoperiodic pathways. New Phytol 199: 1045-1059.

Chen YY, Wang Y, Shin LJ, Wu JF, Shanmugam V, Tsednee M, Lo JC, Chen CC, Wu SH, Yeh KC. 2013. Iron is involved in the maintenance of circadian period length in Arabidopsis. Plant Physiol 161: 1409-1420.

Chow BY, Sanchez SE, Breton G, Pruneda-Paz JL, Krogan NT, Kay SA. 2014. Transcriptional regulation of LUX by CBF1 mediates cold input to the circadian clock in Arabidopsis. Curr Biol 24: 1518-1524.

Covington MF, Harmer SL. 2007. The circadian clock regulates auxin signaling and responses in Arabidopsis. PLoS Biol 5: e222.

Covington M, Maloof J, Straume M, Kay S, Harmer S. 2008. Global transcriptome analysis reveals circadian regulation of key pathways in plant growth and development. Genome Biol 9: R130.

Dalchau N, Baek SJ, Briggs HM, Robertson FC, Dodd AN, Gardner MJ, Stancombe MA, Haydon MJ, Stan GB, Gonçalves JM, et al. 2011. The circadian oscillator gene GIGANTEA mediates a long-term response of the Arabidopsis thaliana circadian clock to sucrose. Proc Natl Acad Sci 108: 5104-5109.

de Lucas M, Prat S. 2014. PIFs get BRright: PHYTOCHROME INTERACTING FACTORs as integrators of light and hormonal signals. New Phytol 202: 1126-1141.

Ding Z, Millar AJ, Davis AM, Davis SJ. 2007. TIME FOR COFFEE encodes a nuclear regulator in the Arabidopsis thaliana circadian clock. Plant Cell 19: 1522-1536. 
Dodd AN, Salathia N, Hall A, Kevei E, Toth R, Nagy F, Hibberd JM, Millar AJ, Webb AAR. 2005. Plant circadian clocks increase photosynthesis, growth, survival, and competitive advantage. Science 309: 630-633.

Dodd AN, Gardner MJ, Hotta CT, Hubbard KE, Dalchau N, Love J, Assie JM, Robertson FC, Jakobsen MK, Gonçalves $\mathrm{J}$, et al. 2007. The Arabidopsis circadian clock incorporates a cADPR-based feedback loop. Science 318: 1789-1792.

Dodd AN, Kudla J, Sanders D. 2010. The language of calcium signaling. Annu Rev Plant Biol 61: 593-620.

Dodd AN, Belbin FE, Frank A, Webb AAR. 2015. Interactions between circadian clocks and photosynthesis for the temporal and spatial coordination of metabolism. Front Plant Sci 6: 245.

Dong MA, Farré EM, Thomashow MF. 2011. CIRCADIAN CLOCK-ASSOCIATED 1 and LATE ELONGATED HYPOCOTYL regulate expression of the C-REPEAT BINDING FACTOR (CBF) pathway in Arabidopsis. Proc Natl Acad Sci 108: 7241-7246.

Duc C, Cellier F, Lobréaux S, Briat JF, Gaymard F. 2009. Regulation of iron homeostasis in Arabidopsis thaliana by the clock regulator time for coffee. J Biol Chem 284: 36271-36281.

Endo M, Shimizu H, Nohales MA, Araki T, Kay SA. 2014. Tissue-specific clocks in Arabidopsis show asymmetric coupling. Nature 515: 419-422.

Farré EM, Liu T. 2013. The PRR family of transcriptional regulators reflects the complexity and evolution of plant circadian clocks. Curr Opin Plant Biol 16: 621-629.

Farré EM, Harmer SL, Harmon FG, Yanovsky MJ, Kay SA. 2005. Overlapping and distinct roles of $P R R 7$ and $P R R S$ in the Arabidopsis circadian clock. Curr Biol 15: 47-54.

Fogelmark K, Troein C. 2014. Rethinking transcriptional activation in the Arabidopsis circadian clock. PLoS Comput Biol 10: e1003705.

Fukushima A, Kusano M, Nakamichi N, Kobayashi M, Hayashi N, Sakakibara H, Mizuno T, Saito K. 2009. Impact of clock-associated Arabidopsis pseudo-response regulators in metabolic coordination. Proc Natl Acad Sci 106: 7251-7256.

Gayomba SR, Zhai Z, Jung HI, Vatamaniuk OK. 2015. Local and systemic signaling of iron status and its interactions with homeostasis of other essential elements. Front Plant Sci 6: 716.

Gendron JM, Pruneda-Paz JL, Doherty CJ, Gross AM, Kang SE, Kay SA. 2012. Arabidopsis circadian clock protein, TOC1, is a DNA-binding transcription factor. Proc Natl Acad Sci 109: 3167-3172.

Goodspeed D, Chehab EW, Min-Venditti A, Braam J, Covington MF. 2012. Arabidopsis synchronizes jasmonatemediated defense with insect circadian behavior. Proc Natl Acad Sci 109: 4674-4677.

Goodspeed D, Chehab EW, Covington MF, Braam J. 2013. Circadian control of jasmonates and salicylates. Plant Signal Behav 8: e23123.

Graf A, Schlereth A, Stitt M, Smith AM. 2010. Circadian control of carbohydrate availability for growth in Arabidopsis plants at night. Proc Natl Acad Sci 107: 9458-9463.

Greenham K, McClung CR. 2015. Integrating circadian dynamics with physiological processes in plants. Nat Rev Genet 16: 598-610.
Grundy J, Stoker C, Carre IA. 2015. Circadian regulation of abiotic stress tolerance in plants. Front Plant Sci 6: 648.

Gutiérrez RA, Stokes TL, Thum K, Xu X, Obertello M, Katari MS, Tanurdzic M, Dean A, Nero DC, McClung CR, et al. 2008. Systems approach identifies an organic nitrogen-responsive gene network that is regulated by the master clock control gene CCA1. Proc Natl Acad Sci 105: 4939-4944.

Hall A, Bastow RM, Davis SJ, Hanano S, McWatters HG, Hibberd V, Doyle MR, Sung S, Halliday KJ, Amasino RM, et al. 2003. The TIME FOR COFFEE gene maintains the amplitude and timing of Arabidopsis circadian clocks. Plant Cell 15: 2719-2729.

Hanano S, Domagalska MA, Nagy F, Davis SJ. 2006. Multiple phytohormones influence distinct parameters of the plant circadian clock. Genes Cells 11: 1381-1392.

Harmer SL. 2009. The circadian system in higher plants. Annu Rev Plant Biol 60: 357-377.

Harmer SL, Hogenesch JB, Straume M, Chang HS, Han B, Zhu T, Wang X, Kreps JA, Kay SA. 2000. Orchestrated transcription of key pathways in Arabidopsis by the circadian clock. Science 290: 2110-2113.

Hassidim M, Yakir E, Fradkin D, Hilman D, Kron I, Keren N, Harir Y, Yerushalmi S, Green RM. 2007. Mutations in CHLOROPLAST RNA BINDING provide evidence for the involvement of the chloroplast in the regulation of the circadian clock in Arabidopsis. Plant J 51: 551-562.

Haydon MJ, Bell LJ, Webb AAR. 2011. Interactions between plant circadian clocks and solute transport. J Exp Bot 62: 2333-2348.

Haydon MJ, Mielczarek O, Robertson FC, Hubbard KE, Webb AAR. 2013. Photosynthetic entrainment of the Arabidopsis thaliana circadian clock. Nature 502: 689692.

Haydon MJ, Román Á, Arshad W. 2015. Nutrient homeostasis within the plant circadian network. Front Plant Sci 6: 299.

Hazen SP, Schultz TF, Pruneda-Paz JL, Borevitz JO, Ecker JR, Kay SA. 2005. LUX ARRHYTHMO encodes a Myb domain protein essential for circadian rhythms. Proc Natl Acad Sci 102: 10387-10392.

Hong S, Kim SA, Guerinot ML, McClung CR. 2013. Reciprocal interaction of the circadian clock with the iron homeostasis network in Arabidopsis. Plant Physiol 161: 893-903.

Hsu PY, Harmer SL. 2014. Wheels within wheels: The plant circadian system. Trends Plant Sci 19: 240-249.

Hsu PY, Devisetty UK, Harmer SL. 2013. Accurate timekeeping is controlled by a cycling activator in Arabidopsis. eLife 2: $\mathrm{e} 00473$.

Huang W, Pérez-García P, Pokhilko A, Millar AJ, Antoshechkin I, Riechmann JL, Mas P. 2012. Mapping the core of the Arabidopsis circadian clock defines the network structure of the oscillator. Science 336: 75-79.

Huot B, Yao J, Montgomery BL, He SY. 2014. Growth-defense tradeoffs in plants: A balancing act to optimize fitness. Mol Plant 7: 1267-1287.

Ingle RA, Stoker C, Stone W, Adams N, Smith R, Grant M, Carré I, Roden LC, Denby KJ. 2015. Jasmonate signalling drives time-of-day differences in susceptibility of Arabi- 
dopsis to the fungal pathogen Botrytis cinerea. Plant $J$ 84: 937-948.

James AB, Monreal JA, Nimmo GA, Kelly CL, Herzyk P, Jenkins GI, Nimmo HG. 2008. The circadian clock in Arabidopsis roots is a simplified slave version of the clock in shoots. Science 322: 1832-1835.

Johnson CH, Knight MR, Kondo T, Masson P, Sedbrook J, Haley A, Trewavas A. 1995. Circadian oscillations of cytosolic and chloroplastic free calcium in plants. Science 269: $1863-1865$.

Kazan K, Lyons R. 2015. The link between flowering time and stress tolerance. J Exp Bot 67: 47-60.

Kim WY, Ali Z, Park HJ, Park SJ, Cha J-Y, Perez-Hormaeche J, Quintero FJ, Shin G, Kim MR, Qiang Z, et al. 2013a. Release of SOS2 kinase from sequestration with GIGANTEA determines salt tolerance in Arabidopsis. Nat Commun 4: 1352.

Kim Y, Han S, Yeom M, Kim H, Lim J, Cha JY, Kim WY, Somers David E, Putterill J, Nam Hong G, et al. 2013b. Balanced nucleocytosolic partitioning defines a spatial network to coordinate circadian physiology in plants. Dev Cell 26: 73-85.

Knight H, Thomson AJW, McWatters HG. 2008. SENSITIVE TO FREEZING6 integrates cellular and environmental inputs to the plant circadian clock. Plant Physiol 148: $293-303$.

Kolmos E, Chow BY, Pruneda-Paz JL, Kay SA. 2014. HsfB2bmediated repression of PRR7 directs abiotic stress responses of the circadian clock. Proc Natl Acad Sci 111: 16172-16177.

Larrieu A, Vernoux T. 2015. Comparison of plant hormone signalling systems. Essays Biochem 58: 165-181.

Lee KH, Piao HL, Kim HY, Choi SM, Jiang F, Hartung W, Hwang I, Kwak JM, Lee IJ, Hwang I. 2006. Activation of glucosidase via stress-induced polymerization rapidly increases active pools of abscisic acid. Cell 126: 1109-1120.

Legnaioli T, Cuevas J, Mas P. 2009. TOC1 functions as a molecular switch connecting the circadian clock with plant responses to drought. EMBO J 28: 3745-3757.

Liu T, Carlsson J, Takeuchi T, Newton L, Farré EM. 2013. Direct regulation of abiotic responses by the Arabidopsis circadian clock component PRR7. Plant J 76: 101-114.

Love J, Dodd AN, Webb AAR. 2004. Circadian and diurnal calcium oscillations encode photoperiodic information in Arabidopsis. Plant Cell 16: 956-966.

Lu SX, Knowles SM, Andronis C, Ong MS, Tobin EM. 2009. CIRCADIAN CLOCK ASSOCIATED1 and LATE ELONGATED HYPOCOTYL function synergistically in the circadian clock of Arabidopsis. Plant Physiol 150: 834-843.

Lu SX, Webb CJ, Knowles SM, Kim SHJ, Wang Z, Tobin EM. 2012. CCA1 and ELF3 interact in the control of hypocotyl length and flowering time in Arabidopsis. Plant Physiol 158: $1079-1088$.

McClung CR. 2013. Beyond Arabidopsis: The circadian clock in non-model plant species. Semin Cell Dev Biol 24: 430436.

Meinke DW, Cherry JM, Dean C, Rounsley SD, Koornneef M. 1998. Arabidopsis thaliana: A model plant for genome analysis. Science 282: 662-682.

Michael TP, Salomé PA, Yu HJ, Spencer TR, Sharp EL, McPeek MA, Alonso JM, Ecker JR, McClung CR. 2003.
Enhanced fitness conferred by naturally occurring variation in the circadian clock. Science 302: 1049-1053.

Mizuno T, Yamashino T. 2008. Comparative transcriptome of diurnally oscillating genes and hormone-responsive genes in Arabidopsis thaliana: Insight into circadian clock-controlled daily responses to common ambient stresses in plants. Plant Cell Physiol 49: 481-487.

Mohawk JA, Green CB, Takahashi JS. 2012. Central and peripheral circadian clocks in mammals. Annu Rev Neurosci 35: 445-462.

Müller NA, Wijnen CL, Srinivasan A, Ryngajllo M, Ofner I, Lin T, Ranjan A, West D, Maloof JN, Sinha NR, et al. 2015. Domestication selected for deceleration of the circadian clock in cultivated tomato. Nat Genet 48: 89-93.

Nagel DH, Kay SA. 2012. Complexity in the wiring and regulation of plant circadian networks. Curr Biol 22: R648-R657.

Nagel DH, Pruneda-Paz JL, Kay SA. 2014. FBH1 affects warm temperature responses in the Arabidopsis circadian clock. Proc Natl Acad Sci 111: 14595-14600.

Nagel DH, Doherty CJ, Pruneda-Paz JL, Schmitz RJ, Ecker JR, Kay SA. 2015. Genome-wide identification of CCA1 targets uncovers an expanded clock network in Arabidopsis. Proc Natl Acad Sci 112: E4802-E4810.

Nakamichi N, Kita M, Ito S, Sato E, Yamashino T, Mizuno T. 2005. The Arabidopsis pseudo-response regulators, PRR5 and PRR7, coordinately play essential roles for circadian clock function. Plant Cell Physiol 46: 609-619.

Nakamichi N, Kusano M, Fukushima A, Kita M, Ito S, Yamashino T, Saito K, Sakakibara H, Mizuno T. 2009. Transcript profiling of an Arabidopsis PSEUDO RESPONSE REGULATOR arrhythmic triple mutant reveals a role for the circadian clock in cold stress response. Plant Cell Physiol 50: 447-462.

Nakamichi N, Kiba T, Henriques R, Mizuno T, Chua NH, Sakakibara H. 2010. PSEUDO-RESPONSE REGULATORS 9, 7, and 5 are transcriptional repressors in the Arabidopsis circadian clock. Plant Cell 22: 594-605.

Nakamichi N, Kiba T, Kamioka M, Suzuki T, Yamashino T, Higashiyama T, Sakakibara H, Mizuno T. 2012. Transcriptional repressor PRR5 directly regulates clock-output pathways. Proc Natl Acad Sci 109: 17123-17128.

Nolte C, Staiger D. 2015. RNA around the clock. Front Plant Sci 6: 311 .

Noordally ZB, Ishii K, Atkins KA, Wetherill SJ, Kusakina J, Walton EJ, Kato M, Azuma M, Tanaka K, Hanaoka M, et al. 2013. Circadian control of chloroplast transcription by a nuclear-encoded timing signal. Science 339: 13161319.

Nováková M, Motyka V, Dobrev PI, Malbeck J, Gaudinová A, Vanková R. 2005. Diurnal variation of cytokinin, auxin and abscisic acid levels in tobacco leaves. J Exp Bot 56: 2877-2883.

Nozue K, Covington M, Duek P, Lorrain S, Fankhauser C, Harmer S, Maloof J. 2007. Rhythmic growth explained by coincidence between internal and external cues. Nature 19: $358-361$.

Nusinow DA, Helfer A, Hamilton EE, King JJ, Imaizumi T, Schultz TF, Farre EM, Kay SA. 2011. The ELF4-ELF3LUX complex links the circadian clock to diurnal control of hypocotyl growth. Nature 475: 398-402. 
Okada R, Kondo S, Satbhai SB, Yamaguchi N, Tsukuda M, Aoki S. 2009. Functional characterization of CCA1/LHY homolog genes, PpCCA1a and PpCCA1b, in the moss Physcomitrella patens. Plant J 60: 551-563.

Panigrahi KCS, Mishra P. 2015. GIGANTEA—An emerging story. Front Plant Sci 6: 8.

Park DH, Somers D, Kim JS, Choy YH, Lim HK, Soh MS, Kim HJ, Kay SA, Nam HG. 1999. Control of circadian rhythms and photoperiodic flowering by the Arabidopsis GIGANTEA gene. Science 285: 1579-1582.

Peñarrubia L, Romero P, Carrió-Seguí A, Andrés-Bordería A, Moreno J, Sanz A. 2015. Temporal aspects of copper homeostasis and its crosstalk with hormones. Front Plant Sci 6: 255.

Perea-García A, Andrés-Colás N, Peñarrubia L. 2010. Copper homeostasis influences the circadian clock in Arabidopsis. Plant Signal Behav 5: 1237-1240.

Perea-García A, Andrés-Bordería A, Mayo de Andrés S, Sanz A, Davis AM, Davis SJ, Huijser P, Peñarrubia L. 2015. Modulation of copper deficiency responses by diurnal and circadian rhythms in Arabidopsis thaliana. J Exp Bot 67: 391-403.

Pérez-García P, Ma Y, Yanovsky MJ, Mas P. 2015. Time-dependent sequestration of RVE8 by LNK proteins shapes the diurnal oscillation of anthocyanin biosynthesis. Proc Natl Acad Sci 112: 5249-5253.

Pilgrim ML, McClung CR. 1993. Differential involvement of the circadian clock in the expression of genes required for ribulose-1,5-bisphosphate carboxylase/oxygenase synthesis, assembly, and activation in Arabidopsis thaliana. Plant Physiol 103: 553-564.

Pokhilko A, Fernandez AP, Edwards KD, Southern MM, Halliday KJ, Millar AJ. 2012. The clock gene circuit in Arabidopsis includes a repressilator with additional feedback loops. Mol Syst Biol 8: 574.

Pruneda-Paz JL, Kay SA. 2010. An expanding universe of circadian networks in higher plants. Trends Plant Sci 15: 259-265.

Ravet K, Touraine B, Boucherez J, Briat JF, Gaymard F, Cellier F. 2009. Ferritins control interaction between iron homeostasis and oxidative stress in Arabidopsis. Plant J 57: $400-412$.

Rawat R, Takahashi N, Hsu PY, Jones MA, Schwartz J, Salemi MR, Phinney BS, Harmer SL. 2011. REVEILLE8 and PSEUDO-REPONSE REGULATOR5 form a negative feedback loop within the Arabidopsis circadian clock. PLoS Genet 7: e1001350.

Robertson F, Skeffington A, Gardner M, Webb A. 2009. Interactions between circadian and hormonal signalling in plants. Plant Mol Biol 69: 419-427.

Romanowski A, Yanovsky MJ. 2015. Circadian rhythms and post-transcriptional regulation in higher plants. Front Plant Sci 6: 437.

Ruffel S, Krouk G, Coruzzi GM. 2010. A systems view of responses to nutritional cues in Arabidopsis: Toward a paradigm shift for predictive network modeling. Plant Physiol 152: 445-452.

Rugnone ML, Faigón Soverna A, Sanchez SE, Schlaen RG, Hernando CE, Seymour DK, Mancini E, Chernomoretz A, Weigel D, Más P, et al. 2013. LNK genes integrate light and clock signaling networks at the core of the Arabidopsis oscillator. Proc Natl Acad Sci 110: 12120-12125.

Salomé PA, Oliva M, Weigel D, Krämer U. 2013. Circadian clock adjustment to plant iron status depends on chloroplast and phytochrome function. EMBO J 32: 511523.

Sánchez JP, Duque P, Chua NH. 2004. ABA activates ADPR cyclase and cADPR induces a subset of ABA-responsive genes in Arabidopsis. Plant J 38: 381-395.

Sanchez SE, Petrillo E, Beckwith EJ, Zhang X, Rugnone ML, Hernando CE, Cuevas JC, Godoy Herz MA, DepetrisChauvin A, Simpson CG, et al. 2010. A methyl transferase links the circadian clock to the regulation of alternative splicing. Nature 468: 112-116.

Sanchez-Villarreal A, Shin J, Bujdoso N, Obata T, Neumann U, Du SX, Ding Z, Davis AM, Shindo T, Schmelzer E, et al. 2013. TIME FOR COFFEE is an essential component in the maintenance of metabolic homeostasis in Arabidopsis thaliana. Plant J 76: 188-200.

Seo PJ, Mas P. 2014. Multiple layers of posttranslational regulation refine circadian clock activity in Arabidopsis. Plant Cell 26: 79-87.

Seo PJ, Mas P. 2015. STRESSing the role of the plant circadian clock. Trends Plant Sci 20: 230-237.

Seung D, Risopatron J, Jones B, Marc J. 2012. Circadian clock-dependent gating in ABA signalling networks. Protoplasma 249: 445-457.

Shin J, Heidrich K, Sanchez-Villarreal A, Parker JE, Davis SJ. 2012. TIME FOR COFFEE represses accumulation of the MYC2 transcription factor to provide time-of-day regulation of jasmonate signaling in Arabidopsis. Plant Cell 24: $2470-2482$.

Smith AM, Stitt M. 2007. Coordination of carbon supply and plant growth. Plant Cell Environ 30: 1126-1149.

Smith JL, De Moraes CM, Mescher MC. 2009. Jasmonateand salicylate-mediated plant defense responses to insect herbivores, pathogens and parasitic plants. Pest Manag Sci 65: 497-503.

Takahashi N, Hirata Y, Aihara K, Mas P. 2015. A hierarchical multi-oscillator network orchestrates the Arabidopsis circadian system. Cell 163: 148-159.

Thain SC, Vandenbussche F, Laarhoven LJJ, Dowson-Day MJ, Wang ZY, Tobin EM, Harren FJM, Millar AJ, Van Der Straeten D. 2004. Circadian rhythms of ethylene emission in Arabidopsis. Plant Physiol 136: 37513761.

Velez-Ramirez AI, van Ieperen W, Vreugdenhil D, Millenaar FF. 2011. Plants under continuous light. Trends Plant Sci 16: $310-318$.

Velez-Ramirez AI, van Ieperen W, Vreugdenhil D, van Poppel PMJA, Heuvelink E, Millenaar FF. 2014. A single locus confers tolerance to continuous light and allows substantial yield increase in tomato. Nat Commun 5: 4549.

Wang W, Barnaby JY, Tada Y, Li H, Tor M, Caldelari D, Lee DU, Fu XD, Dong X. 2011. Timing of plant immune responses by a central circadian regulator. Nature $\mathbf{4 7 0}$ : $110-114$.

Wang G, Zhang C, Battle SL, Lu H. 2014. The phosphate transporter PHT4;1 is a salicylic acid regulator likely 
S.E. Sanchez and S.A. Kay

controlled by the circadian clock protein CCA1. Front Plant Sci 5: 701.

Xie Q, Wang P, Liu X, Yuan L, Wang L, Zhang C, Li Y, Xing H, Zhi L, Yue Z, et al. 2014. LNK1 and LNK2 are transcriptional coactivators in the Arabidopsis circadian oscillator. Plant Cell 26: 2843-2857.

Xu G, Fan X, Miller AJ. 2012. Plant nitrogen assimilation and use efficiency. Annu Rev Plant Biol 63: 153-182.

Yakir E, Hilman D, Kron I, Hassidim M, Melamed-Book N, Green RM. 2009. Posttranslational regulation of CIRCADIAN CLOCK ASSOCIATED1 in the circadian oscillator of Arabidopsis. Plant Physiol 150: 844-857.
Zhang N, Kallis RP, Ewy RG, Portis AR. 2002. Light modulation of Rubisco in Arabidopsis requires a capacity for redox regulation of the larger Rubisco activase isoform. Proc Natl Acad Sci 99: 3330-3334.

Zheng XY, Zhou M, Yoo H, Pruneda-Paz JL, Spivey NW, Kay SA, Dong X. 2015. Spatial and temporal regulation of biosynthesis of the plant immune signal salicylic acid. Proc Natl Acad Sci 112: 9166-9173.

Zhou M, Wang W, Karapetyan S, Mwimba M, Marques J, Buchler NE, Dong X. 2015. Redox rhythm reinforces the circadian clock to gate immune response. Nature 523: $472-476$. 


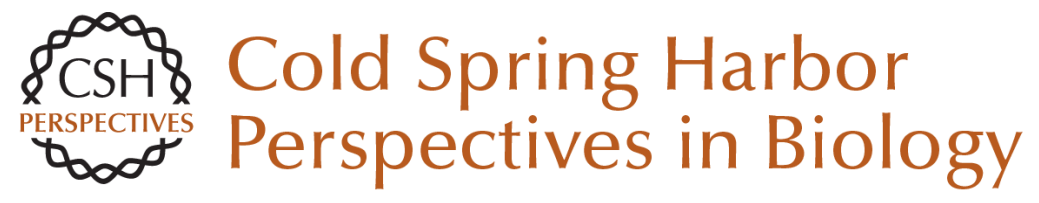

\title{
The Plant Circadian Clock: From a Simple Timekeeper to a Complex Developmental Manager
}

\author{
Sabrina E. Sanchez and Steve A. Kay
}

Cold Spring Harb Perspect Biol 2016; doi: 10.1101/cshperspect.a027748 originally published online September 23, 2016

\section{Subject Collection Circadian Rhythms}

\section{Circadian Posttranscriptional Regulatory \\ Mechanisms in Mammals \\ Carla B. Green}

Design Principles of Phosphorylation-Dependent

Timekeeping in Eukaryotic Circadian Clocks

Koji L. Ode and Hiroki R. Ueda

Interplay between Microbes and the Circadian

Clock

Paola Tognini, Mari Murakami and Paolo Sassone-Corsi

A 50-Year Personal Journey: Location, Gene

Expression, and Circadian Rhythms Michael Rosbash

Regulating the Suprachiasmatic Nucleus (SCN)

Circadian Clockwork: Interplay between

Cell-Autonomous and Circuit-Level Mechanisms Erik D. Herzog, Tracey Hermanstyne, Nicola J. Smyllie, et al.

Systems Chronobiology: Global Analysis of Gene Regulation in a 24-Hour Periodic World Jérôme Mermet, Jake Yeung and Felix Naef
Coordination between Differentially Regulated

Circadian Clocks Generates Rhythmic Behavior Deniz Top and Michael W. Young

Introduction to Chronobiology Sandra J. Kuhlman, L. Michon Craig and Jeanne F. Duffy

Cellular Timekeeping: It's Redox o'Clock Nikolay B. Milev, Sue-Goo Rhee and Akhilesh B. Reddy

Molecular Mechanisms of Sleep Homeostasis in Flies and Mammals

Ravi Allada, Chiara Cirelli and Amita Sehgal

Membrane Currents, Gene Expression, and Circadian Clocks

Charles N. Allen, Michael N. Nitabach and Christopher S. Colwell

The Plant Circadian Clock: From a Simple Timekeeper to a Complex Developmental Manager Sabrina E. Sanchez and Steve A. Kay

For additional articles in this collection, see http://cshperspectives.cshlp.org/cgi/collection/

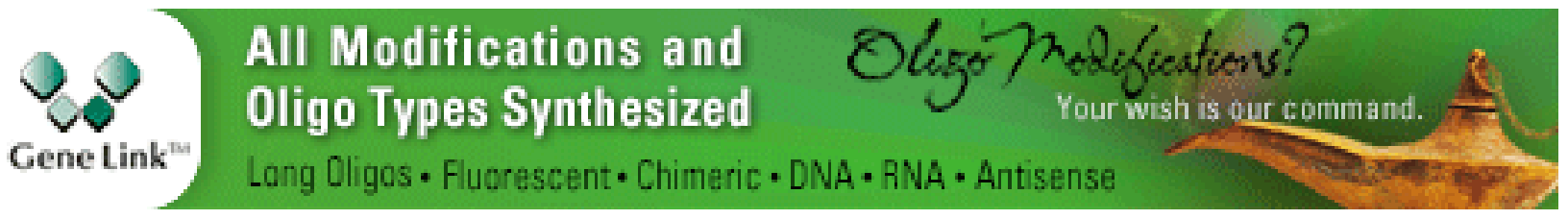

